Alessandro Baratta

\title{
Kriminalpolitik und Verfassung
}

Überlegungen zum minimalen Strafrecht und zur Sicherheit der Rechte

»Kriminalpolitik « ist ein voraussetzungsreicher Begriff. Nicht nur weil ihre praktischen und theoretischen Modelle multiple Bestimmungen aufweisen, sondern vor allem, weil ihre Elemente mit den Grundbegriffen des Staats und der Politik eng verknüpft sind. Die stets aktuelle Frage nach der Fähigkeit der Kriminalpolitik, die Rechte aller effektiv zu schützen, impliziert eine »Rekonstruktion « dieses Begriffs. Nach einer Darstellung der Probleme, die mit den gängigen Definitionen der Kriminalpolitik verbunden sind, werden wir auf die Problematik der Sicherheit der Rechte und auf die epistemologischen Fragen der Konstruktion einer Kriminalpolitik eingehen. Dies wird uns erlauben, die Bedingungen eines Übergangs von der Kriminalpolitik zu einer Politik des Schutzes der Menschenrechte aufzuzeigen.

\section{Kriminalpolitik: ein komplexer und problematischer Begriff}

Ein Grundmerkmal der Kriminalpolitik ist die Komplexität ${ }^{1}$ ihrer Zwecke und Instrumente. Dem traditionellen Verständnis zur Folge hat die Kriminalpolitik für die Kontrolle der Kriminalität zu sorgen; ihre Aufgabe wird in der Verminderung der Zahl der Verstöße gegen die Strafrechtsnormen gesehen ${ }^{2}$. Das weiter anwachsende Interesse für die Bedürfnisse der Opfer und ihrer sozialen Umgebung trägt zu einer Vergrößerung des Aktionsradius der Kriminalpolitik bei: Den Präventionszwecken gesellt sich nun die Sorge um die Kontrolle der Konsequenzen von Kriminalität hinzu ${ }^{3}$.

Hinsichtlich der Instrumente zur Kontrolle der Delikte und ihrer Konsequenzen wird die Ambivalenz noch größer, weil die Konstruktion der Modelle zugleich eine Auswahl der relevanten Variablen setzt, die vom jeweils vertretenen theoretischen Standpunkt abhängig sind. Somit entsteht eine theoretische Klimax: In den unteren Stufen finden sich die Modelle der ätiologischen Kriminologie, die allein auf die Kontrolle der Kriminalität anwendbar sind. Auf mittlerer Ebene stehen jene ätiologischen

1 S. etwa Delmas-Marty, 1983 und 1986; Lazerges, 1987.

2 Gassin, 1988, 359 und 516; Stefani, Levasseur, Bouloc, 1992, 17. Eine weite und angemessenere Definition geben Delmas-Marty, 1986, 123; Lazerges, 1987, 6-7.

3 Zur Debatte und zu den gesetzlichen Regelungen in vielen europäischen Ländern s. Delmas-Marty, 1986, 27 und 61-68; Lazerges, 1987, 71-75; Zauberman, 1991; Roché, 1995; Baratta, 1988, 528; Pepino, Scatolero, 1992; Scatolero, 1992; Krauss, 1983; Kaiser, 1985, 109-113; Schünemann, 1986; Jung, 1987, Frommel 1993, 257-268. Diese Entwicklung steht in Verbindung mit den Forschungen der Strafrechtslehre in bezug auf die Folgen der Kriminalität und der gerichtlichen Entscheidungen; dazu Baratta, 1998, 139 ff. 
Modelle, die auch eine Kontrolle der Konsequenzen der Delinquenz beabsichtigen. Die obere Ebene belegen die Modelle der kritischen Kriminologie, die den labeling approach adoptieren und somit eine adäquatere Kontrolle der Konsequenzen abweichenden Verhaltens ermöglichen.

Der Begriff der Kriminalpolitik ist jedoch nicht nur komplex, sondern auch problematisch. In ihrem umfassenderen Sinn bezeichnet die Kriminalpolitik eine Menge von Aktionen, die viel komplexer und weiter gefaßt sind als die Spezies der »Strafpolitik «. ${ }^{4}$ Die Trennungslinien zwischen der Politik im allgemeinen und der Kriminalpolitik verschwimmen.

Nehmen wir ein Beispiel: Seit Beginn der 80er Jahre formieren sich in Frankreich Komitees, die aus Vertretern der kommunalen Selbstverwaltung, Polizeibeamten, Vertretern verschiedener Vereine und Gewerkschaften sowie anderen Akteuren zusammengesetzt sind; ihr Ziel ist der Entwurf und die Anwendung von Programmen gegen die Marginalisierung von Jugendlichen gemäß der kriminalpolitischen Vorstellungen der nouvelle prévention, die mit öffentlichen Mitteln finanziert werden ${ }^{5}$.

Die Tätigkeit dieser Komitees unterscheidet sich im wesentlichen nicht von Praktiken, die andere Instanzen unter anderen Etiketten, z.B. dem der sozialpolitischen Maßnahmen zur Erziehung »devianter « Jugendlicher, anwenden. Andererseits hängt die Frage, ob eine bestimmte Politik die Kriminalitätsprävention im Sinne einer Verstärkung der Sicherheit der urbanen Bevölkerung oder im Sinne des Schutzes der Grundrechte von Jugendlichen in schwierigen Situationen beabsichtigt, nicht so sehr mit der objektiven Zielsetzung dieser sozialen Praktiken zusammen, als vielmehr mit den (subjektiven) Intentionen der Akteure, die diese verschiedenen Programme entwerfen und anwenden.

\section{Sicherheit oder Sozialpolitik: eine falsche Alternative}

Eine Differenzierung zwischen Sicherheitspolitik und Sozialpolitik ist nicht logisch, sie ist vielmehr ideologisch. Ihre Anwendung im Bereich der Kriminalpolitik hat besonders schwerwiegende Konsequenzen: Durch das Auseinanderhalten dieser Begriffe wird die ohnehin schon komplexe und problematische Kriminalpolitik zu einem rein ideologischen Instrument ${ }^{6}$, welches ausschließlich der Reproduktion und

4 Im Rahmen einer Analyse verschiedener Modelle der Kriminalpolitik spricht DelmasMarty von einem »Feld« der Kriminalpolitik, welches »zugleich weiter und offener « ist als der strafrechtliche Bereich (1983 und 1986, 30, 123-169).

5 Chevalier, 1988; Robert, Renouard, 1991, 192-193; Lazerges, 1987, 106-112; Robert, 1994, 55-56; Duprez, 1997, 67-80. Es ist bezeichnend, daß in vielen europäischen Ländern die finanziellen Ressourcen, die den Präventionstätigkeiten außerhalb des Strafrechtssystems zugewiesen werden, unverhältnismäßig gering sind im Vergleich zu den Funktionskosten der strafrechtlichen Apparate. Vgl. dazu Creazzo, 1996, 14; Duprez, 1997, 80.

6 Der Terminus »Ideologie« wird hier im Sinne einer diskursiven Konstruktion der gesellschaftlichen Fakten benutzt, die zur Bildung eines falschen Bewußtseins der institutionellen Akteure und der öffentlichen Meinung führt. 
Legitimierung des Bestehenden dient. Versuchen wir eine Annäherung am Beispiel der $\gg$ Sicherheitspolitik «.

Das Substantiv »Sicherheit« wird des öfteren durch ein Adjektiv (über-)determiniert: nationale, öffentliche oder städtische Sicherheit. Dadurch wird die Sicherheit als ausschließlich kollektiver Begriff verstanden; es handelt sich nicht um die Sicherheit der Rechte von Personen, die in verschiedenen sozialen Kontexten leben und agieren, sondern um die Sicherheit »der« Nation, »der« politischen Gemeinschaft oder »der« Stadt.

Die Doktrin der »nationalen Sicherheit« gelangte im Lateinamerika der 70er und 80er Jahre zu trauriger Berühmtheit. Eine autoritäre Staatsideologie machte, um ein »feindorientiertes Strafrecht « zu legitimieren ${ }^{7}$, normativen Gebrauch von Carl Schmitts Analysen (1963) bezüglich des »existentiellen« Kampfes zwischen »Freund und Feind «; die Spuren dieser Doktrin lassen sich selbst in formell demokratischen Staaten (wie z.B. in Brasilien) verfolgen. Parallel dazu führte die Doktrin der nationalen Sicherheit zur Schaffung eines »zweiten« Strafsystems, welches außerhalb der verfassungsmäßigen Legalität operierte und sich als viel »effizienter« erwies. Es handelte sich um einen echten Staatsterrorismus, der vor allem von Diktaturen des »CONOSUR « Lateinamerikas praktiziert wurde ${ }^{8}$.

In anderem Kontext hat der Begriff der öffentlichen Sicherheit (oder der Staatssicherheit) die europäische Strafrechtsgeschichte stark geprägt: er ist hier Ausdruck eines Kompromisses zwischen dem liberalen Denken und der autoritären Tradition (»Obrigkeitsstaat«) bzw. zwischen der Strafrechtspolitik und der Politik der öffentlichen Ordnung? .

Der aktuellere Begriff städtischer Sicherheit ist ein wichtiges Ergebnis der Bewegung der $»$ nouvelle prévention ${ }^{10}$. Er unterscheidet sich dadurch von den beiden anderen, daß er im Rahmen der Kriminalpolitik der lokalen und partizipativen Dimension eine besondere Bedeutung zumißt. In seinem Rahmen werden zahlreiche Disziplinen und Organisationen angesprochen ${ }^{11}$ und eine Überwindung der Abschottung der Institutionen experimentell versucht ${ }^{12}$.

In all diesen Fällen kann man jedoch behaupten, daß die Adjektive das Substantiv Sicherheit »erwürgen«. Aus juristischer Perspektive betrachtet, kann Sicherheit nur ein Attribut sein, welches gleichermaßen allen Subjekten zukommt, die Träger von Menschenrechten sind, d.h. sie ist ein Attribut einer jeden Person, die sich auf dem Territorium eines Staates, einer Stadt oder einer anderen territorialen Einheit befindet.

7 Ferrajoli spricht über das »Paradigma des Feindes«, das zu einem Täterstrafrecht führt (1990, 858-859); vgl. Hannover, 1989.

8 Baratta, Silbernagl, 1988, 40-44; Zaffaroni, 1986; García Mendez, 1985.

$9 \mathrm{Zu}$ einer kritischen Analyse der Bestrafungsmodelle, die sich zwischen dem Notstandsstrafrecht und dem offenen Staatsterrorismus bewegen, s. Ferrajoli, 1990, 844-877.

$10 \mathrm{Zu}$ einer kritischen Darstellung dieser Bewegung s. Chevalier, 1988; Robert, 1991 und 1994; Poulet, Brion, Dupont, 1991; Baratta, 1993b; Creazzo, 1996; Lehne, 1996; Hebberecht, Sack, 1997.

11 Robert, Renouard, 1991, 193; Vourc'h, 1991, 266; Selmini, 1994; Duprez, 1997, 72-80.

12 Delmas-Marty, 1986, 101-102 m. w. Hinw.; Lazerges, 1987, 103-117. 
Die Sicherheit bezieht sich auf die effektive Gewährleistung der Grundrechte gegen jeglichen Eingriff oder gegen unangemessenes Verhalten von Machtträgern im Rahmen einer territorialen Einheit, sei es eine rechtliche oder faktische Form der Machtausübung.

Die Behauptung, daß ein Staat, eine Stadt oder eine Gemeinschaft sicher sind, ist ein abstrakter Ausdruck, der die Situation aller Personen, die sich in diesem Territorium befinden, ausdrücken könnte. In der aktuellen Situation jedoch haben solche abstrakten Aussagen einen rein ideologischen Charakter. Sie hängen von einer Denkweise ab, die sowohl in der öffentlichen Meinung als auch im juristischen Diskurs besonders verbreitet ist. Dort wird der Begriff der Sicherheit im Zusammenhang mit den Begriffen der Kriminalpolitik und der Politik im allgemeinen verwendet. Eine kritische Analyse kann zeigen, daß in diesen Diskursen die meisten Grundrechtsträger und die Mehrheit der »Risikoviertel« keine Sicherheit genießen, d. h. von dem Kalkül der Sicherheit praktisch ausgenommen werden.

Diese Diskurse verbinden nämlich die öffentliche Sicherheit mit einer verhältnismäBig geringen Anzahl öffentlich zugänglicher Orte sowie nur einigen wenigen Delikten, die der sog. traditionellen Kriminalität zugehören (Eingriffe, die die Ausübung physischer Gewalt gegen Personen oder Sachen implizieren) ${ }^{13}$. Diese Akte gehören zum Stereotyp des Kriminellen und dominieren die verschiedenen alarmierenden Kampagnen in bezug auf die »Furcht vor der Kriminalität«. In der öffentlichen Meinung und in den Medien werden, im allgemeinen, diese Eingriffe nach einem Opfer/Täter-Dualismus dargestellt, der weitgehend der gesellschaftlichen Differenzierung zwischen privilegierten und »respektablen« Gruppen einerseits und marginalen und »gefährlichen« Gruppen andererseits entspricht. Als Aggressoren gelten dabei vor allem die Ausländer, die Drogenabhängigen, die Armen, die Obdachlosen oder die einfachen Handarbeiter.

Die Gefahrensituationen, denen Frauen und Kinder in der häuslichen Sphäre ausgesetzt sind, und der Entzug sozialer Rechte, dessen Opfer Personen der marginalisierten und »gefährlichen « Gruppen sind, werden in dem urbanen Sicherheitskalkül und der urbanen Sicherheitspolitik nicht berücksichtigt; sehr oft wird nämlich unter der Etikette »lokaler Prävention « nur eine autoritäre und repressive Politik durchgeführt.

Die ökonomischen und ökologischen Delikte, die Korruption, die kriminellen Abweichungen staatlicher Organe und die Verbindung zwischen politischen Machtträgern und mafiaartigen Organisationen werden öffentlich diskutiert vor allem als ein moralisches Problem und nicht als ein Problem der urbanen Sicherheit. Die Frage der öffentlichen Ordnung stellt sich nämlich nur in den eng umschriebenen Grenzen des Handlungsbereichs der »Sicherheitskräfte« und berücksichtigt höchstens indirekt die soziale und internationale Unordnung, die immer beunruhigender wird unter den kombinierten Effekten des Neoliberalismus und der Globalisierung der Ökonomie (Albrecht 1997, 126-127). So vermehren sich die »außerrechtlichen « Bereiche (Scatolero, 1992, 183) im Herz der europäischen Metropolen. Die Unsicherheit dominiert und sucht vor allem die »schwachen« Gruppen heim.

13 S. u.a. Vourc'h, 1991, 273; Lehne, 1996, 314. 
Die ideologische Ambivalenz des Begriffs »Kriminalpolitik « wird noch eindeutiger, wenn wir seine Beziehungen zum anderen Terminus des scheinbaren Dilemmas untersuchen. Es handelt sich um die Sozialpolitik, die eine Art Kompensation gewähren soll für all das, was gewissen Grundrechtsträgern im Rahmen des Sicherheitskalküls verwehrt wurde. Die Kriminalpolitik »vergißt«, die Sicherheitsrechte der marginalisierten und »gefährlichen« Gruppen zu garantieren, und findet diese dann als »Objekte« der Sozialpolitik wieder. Es handelt sich nunmehr um Objekte und nicht um Subjekte, weil die (subjektive) Zwecksetzung der Hilfsprogramme nicht die Garantie ihrer Rechte, sondern die Verstärkung der Sicherheit ihrer potentiellen Opfer ist.

Die Kriminalpolitik wandelt sich, in der Terminologie der »nouvelle prévention «, zur sozialen Prävention der Kriminalität (Robert 1991a, 16; Creazzo 1996, 23-24), um die »respektablen « Personen vor »Gefahren « zu schützen. Die verletzlichen oder verletzten Personen, die an Läsionen ihrer »schwachen « Rechte leiden (ökonomische und soziale Rechte), werden zu potentiellen Aggressoren der »starken« Rechte (körperliche Integrität, Eigentum) der gesellschaftlich am besten geschützten Personen ${ }^{14}$.

Der Staat versucht, durch die soziale Prävention vor allem seiner Schutzpflicht nachzukommen, indem er die schwächeren Gruppen als Risikofaktoren betrachtet. Und dadurch vernachlässigt er seine Leistungspflicht gegenüber den verletzlichen Personen. In diesem Rahmen stellt sich die Kriminalpolitik über die Sozialpolitik, und führt zur ihrer »Kriminalisierung «. Die Behauptung, daß die Kriminalpolitik zwischen einer Politik der Sicherheit und einer Sozialpolitik zu wählen habe, ist eine falsch verstandene Alternative. Wenn wir uns von diesen Stereotypen und dem ideologischen Verständnis der Begriffe befreien, dann erlaubt uns eine strenge Benutzung der juristischen Konzepte, die enge Verbindung der Sicherheit mit den Grundrechten zu verdeutlichen und somit die Alternative zu verwerfen.

Um dies zu zeigen ist es notwendig, die Merkmale, die Transformationen und die Gründe der heutigen Krise desjenigen Wissensbereichs zu untersuchen, auf den sich die Kriminalpolitik stützt. Es handelt sich um die sog. »gesamte Strafrechtswissenschaft «, die mindestens drei unterschiedliche Wissensmodelle umfaßt.

\section{Die gesamte Strafrechtswissenschaft und die Zukunft der Kriminologie}

Am Anfang des 20. Jahrhunderts etablierte sich die positivistische Kriminologie als eine Theorie des Verbrechens und als eine Theorie der Techniken der Kriminalpolitik. Im Zusammenwirken mit der Strafrechtslehre schuf sie ein erstes Modell der »gesamten Strafrechtswissenschaft« (Baratta 1980).

14 Im Rahmen der zentralen Auseinandersetzung um die unterschiedliche Tragweite und Intensität von verfassungsrechtlich garantierten subjektiven Rechten vgl. die Argumentation (in bezug auf »Rechte im starken Sinn«) bei Dworkin, 1990, 303-326, 433 und (in bezug auf die Unterscheidung von »Prinzipien«, die als »Optimierungsgebote« einen »prima-facie-Charakter « aufweisen, und »Regeln«, die einen »definitiven « Charakter haben) bei Alexy, 1986, 71-122, 454-472; Ferrajoli, 1990, 901-907. 
Nach dem Zweiten Weltkrieg zeichnet sich, anfänglich in den U.S.A. und später in vielen Ländern, eine neue theoretische Perspektive ab, die sich vor allem auf den symbolischen Interaktionismus stützt: der sog. labeling approach. In das Zentrum des wissenschaftlichen Interesses werden nunmehr die Mechanismen sowie die institutionellen und informellen Prozesse der kriminellen Ettiketierung gestellt. Der labeling approach demonstrierte die wissenstheoretische Inkonsistenz des ätiologischen Paradigmas und kritisierte die Hilfsrolle der traditionellen Kriminologie in bezug auf das Strafrecht und die Kriminalpolitik (Baratta, 1980; 1982, 85-98, 159-171).

Das integrierte Modell im Sinne v. Liszt's wird sich später, in den 60er Jahren, in einer tiefgreifenden Krise befinden. In der Perspektive des Etikettierungsansatzes übernimmt die Soziologie der Abweichung keine Hilfsrolle mehr, sondern sie analysiert das Strafrecht von einem externen Standpunkt aus. Sie betrachtet es als einen Forschungsgegenstand, der nicht mit den Kategorien der traditionellen Kriminologie erfaßt werden kann. Die Theorie und die Soziologie des Strafrechts im weiten Sinne umfaßt die informellen Projekte der Kriminalisierung, wie z. B. die Reaktion der öffentlichen Meinung auf die Kriminalität, und erstreckt sich auf die Strafrechtslehre, die sie als konstitutive Instanz des Strafrechts betrachtet.

Das Modell der gesamten Strafrechtswissenschaft, das sich aus der Konvergenz der Strafrechtslehre und -soziologie ergibt, unterscheidet sich von dem in der Liszt 'schen Tradition geschaffenen Modell. Die Einnahme eines externen Standpunkts bedeutet u.a., daß die Verhaltensdefinitionen, die aus den Instanzen des Strafrechtssystems gegeben werden (Gesetzgebung, Lehre, Rechtsprechung, Polizei, öffentliche Meinung), nicht mehr als Ausgangspunkt, sondern als Problem und als Studiengegenstand betrachtet werden.

Auch nach dem Paradigmenwechsel in der Strafrechtslehre und -soziologie folgte der wissenschaftliche Diskurs einer Linie der Interdisziplinarität, die als interne bezeichnet werden kann. Eine solche liegt vor, wenn eine oder mehrere Disziplinen einen einzigen Gegenstand haben (in unserem Fall das Strafrecht) und im Rahmen des eigenen Diskurses Resultate anderer Disziplinen integrieren gemäß ihrer eigenen Auswahl- und Einordnungsregeln. Somit können sie die Hegemonie und die Selbständigkeit ihres eigenen Wissens im Vergleich zu den Ergebnissen anderer Disziplinen aufrechterhalten.

Eine solche Interdisziplinarität führte zur Konstruktion eines zweiten Modells der gesamten Strafrechtswissenschaft. Es handelte sich nunmehr um ein kritisches Modell, das u.a. Resultate der Sozialtheorie und -geschichte, der Sozialpsychologie, der Politikwissenschaft, der Argumentationstheorie und der Sozialethik benutzte. Die Inhalte dieses interdisziplinären Diskurses wurden von der Intervention der kritischen Kriminologie gekennzeichnet; ihre Analysen zur Definition des Verbrechens und zur sozialen Reaktion veränderten den harten Kern des ursprünglichen Diskurses, d. h. des integrierten strafrechtlichen Wissens.

Der wissenschaftliche Diskurs, der gemäß der internen Interdisziplinarität erzeugt wird, ermöglicht eine Form der Kontrolle des Strafrechtssystems, welche gleichermaßen als interne zu bezeichnen ist. Es handelt sich um die formelle Kontrolle der Entsprechung zwischen den repressiven Praktiken und den Prinzipien des liberalen 
Strafrechts (Freiheit, Gleichheit, Legalität), in denen das »Versprechen der Moderne« zum Ausdruck kam.

Wir können dagegen als externe diejenige Form der Kontrolle bezeichnen, die sich auf Prinzipien der materiellen Gerechtigkeit und auf politische Kriterien stützt und die somit auch die externen Effekte des Strafrechtssystems berücksichtigt. Dadurch wird die selektive Bestimmung und Verteidigung der Rechtsgüter ${ }^{15}$ berücksichtigt, die sich durch die strafrechtliche Kontrolle problematischer Situationen und Verhaltensweisen ergibt. Gegenstand der externen wissenschaftlichen Kontrolle ist somit die soziale Kosten-Nutzen-Bilanz, die sich durch die Intervention des Strafrechtssystems ergibt (Baratta, 1993, 410 ff.).

Der wissenschaftliche Diskurs, der eine solche Kontrollfunktion übernehmen kann, hat keinen homogenen Gegenstand, wie es bei der internen Kontrolle der Fall ist. Es wurde häufig bemerkt (z.B. Hess, 1986, 34-35, Robert, 1994, 54), daß die Situationen, die das Strafrechtssystem verwaltet, ein Universum heterogener Ereignisse mit fließenden Grenzen bilden ${ }^{16}$. Der einzige gemeinsame Nenner dieser Situationen ist die Tatsache, daß sie für einen gewissen Zeitraum und in einer bestimmten Gesellschaft den Gegenstand eines strafrechtlichen Eingriffes darstellen. Die soziale Negativität dieser Situationen und die Möglichkeit, sie mit dem Verhalten eines Individuums in Verbindung zu bringen (durch die gesellschaftlichen Mechanismen der Zurechnung), sind dagegen weder gemeinsame noch spezifische Merkmale dieser Situationen. In der Tat sind sie nicht in allen Situationen, die das Strafrecht verwaltet, anzutreffen und sie bezeichnen Verhaltensweisen, die gleichermaßen Gegenstand des Eingriffs anderer Systeme sozialer Kontrolle sein könnten.

Wenn wir uns von der Theorie des »natürlichen Verbrechens« distanzieren, die die Kriminalität als eine ontische Eigenschaft bestimmter Verhaltensweisen und Subjekte darstellt, d. h. sie als unabhängig von sozialen Mechanismen der Definition und der Zurechnung der Verantwortung betrachtet ${ }^{17}$, dann müssen wir die Heterogenität und die fließenden Grenzen der kriminalisierten Verhaltensweisen anerkennen. Chapman bemerkte, daß es für fast alle als kriminell definierten Handlungen vergleichbare Verhaltensweisen gibt, die nicht als Gegenstand einer solchen Definition dienen (1968, 174-178). Darüber hinaus müssen wir die Tatsache berücksichtigen, daß das Strafrechtssystem, parallel zu anderen Systemen der präventiven oder repressiven Kontrolle, bestimmte problematische Situationen kontrolliert und daß die Ausschließlichkeits- oder Vorrangsansprüche eines dieser Systeme, ausgehend von der Natur der problematischen Situationen, nicht gerechtfertigt werden kann.

$15 \mathrm{Zu}$ einer kritischen Diskussion des Begriffs und seiner symbolischen und instrumentellen Rolle im Rahmen der Kriminalpolitik s. Baratta, 1993; Smaus 1985.

16 Ist es z.B. möglich, die Formen der »traditionellen« Kriminalität (Delikte gegen das Eigentum, die körperliche Unversehrtheit und die Ehre der Person) als homogen mit den Phänomenen der »neuen « Kriminalität in postindustriellen Gesellschaften zu betrachten (ökologische Delikte, Korruption, technologische Risiken, Drogenhandel, Unsicherheit in den öffentlichen Transportmitteln oder HIV-Infizierung)?

17 Zur Kritik der ontologischen Konzeptionen der Kriminalität s. Baratta, 1982, 32-43, 83129; 1998, $138 \mathrm{ff}$. 
Wenn wir versuchen, die aktuellen Politiken der Kontrolle der sozialen Negativität zu evaluieren und neue Politiken zu entwickeln, so erlaubt uns die Bezugnahme auf das Strafrechtssystem keine zufriedenstellende Definition der »kriminellen« Verhaltensweisen: sie ist nicht spezifisch und sie erscheint nicht als ontisch notwendig. Eine solche Bezugnahme kann sich als nützlich erweisen für die Studie der gesellschaftlichen Prozesse der Definition von Kriminalität zum Zwecke der internen Kontrolle des Strafrechtssystems; sie ist jedoch für die Erarbeitung der Variablen einer externen Kontrolle ungeeignet.

Der Diskurs der zeitgenössischen Kriminologie beinhaltet zwei wichtige Dimensionen: die Dimension der Definition der Delinquenz (oder der sozialen Reaktion) und die Dimension des Verhaltens. Im gegenwärtigen Stand der Diskussion betreffen die meisten Probleme die Dimension des Verhaltens.

In ihrer Dimension der Definition der Delinquenz trägt die kritische Kriminologie als eine Theorie und Soziologie des Strafrechts zur Realisierung eines integrierten Modells der Strafrechtswissenschaft bei. Dieses Modell hat die Funktion, das Strafjustizsystem intern zu kontrollieren. In dieser Dimension beziehen sich die Theorie und die Soziologie des Strafrechts im Rahmen des integrierten Diskurses der Strafrechtswissenschaft, in den sie sich einordnen, auf einen Gegenstand, der ihre Autonomie und ihre Möglichkeit, Elemente anderer Wissensbereiche zu selektieren und im Inneren des eigenen Diskurses zu organisieren (interne Interdisziplinarität), epistemologisch rechtfertigt.

In der Dimension des Verhaltens dagegen ist Gegenstand des Diskurses der materielle Träger der Kriminalität, also die problematische Situation oder die Verletzung von Grundrechten, die auf das Verhalten von Individuen bezogen werden kann. Hier befaßt sich die Kriminologie und der integrierte Diskurs der Strafrechtswissenschaft mit heterogenen problematischen Situationen, um das System extern zu kontrollieren. Damit hören aber die wissenschaftliche Selbständigkeit und die »integrierende « Kompetenz auf. Jeder problematische Bereich verlangt eine Behandlung seitens mehrerer Disziplinen und spezifischer Wissensbereiche, wobei jedoch keine Disziplin im vornherein eine hegemoniale Rolle oder eine Kompetenz-Kompetenz über die Rolle der anderen beanspruchen kann.

Solche Definitionsschwierigkeiten beeinflussen direkt oder indirekt die heutige Debatte über die »Zukunft« der Kriminologie und haben große epistemologische Fragen aufgeworfen, die noch nicht beantwortet sind und die sich im Zentrum einer Krise (einer Entwicklungskrise?) der kritischen Kriminologie befinden ${ }^{18}$.

Wir können als externe Interdisziplinarität eine Form der Konvergenz wissenschaftlicher Disziplinen und spezifischer Wissensbereiche bezeichnen, die dem Prinzip der paritätischen Kompetenz folgt ${ }^{19}$. Die Artikulation der verschiedenen Wissenselemente variiert nicht nur gemäß der Natur der Probleme, die der wissenschaftliche Diskurs zu

18 Nelken, 1994; Swaaningen, 1994; Hess, Scheerer, 1997.

19 Solche Formen der Interdisziplinarität werden von der modernen Epistemologie als verheiBungsvoll betrachtet und oft als »Transdisziplinarität« betrachtet (Gräfrath, Huber, Uhlemann, 1991, 182-185; Mittelstrass 1989). 
kontrollieren versucht, sondern auch gemäß der Dynamik jedes Wissensbereichs und der Perzeption der Probleme durch jene Akteure, die die politische Verantwortung für die Erarbeitung von Kontrollstrategien und -taktiken tragen.

Wir können somit die Hypothese aufstellen, daß die externe Kontrolle des Strafrechtssystems und die Anwendung einer kohärenten Politik bezüglich der sozial problematischen Situationen, die sich auf das Verhalten von Individuen bezieht, den Beitrag einer neuen gesamten Strafrechtswissenschaft verlangen, welche die Form eines differenzierten und beweglichen Netzes von konvergierenden Wissensmengen annimmt und sich gemäß der Regeln der externen Interdisziplinarität konstruiert.

Meiner Meinung nach gibt es keine Zukunft für eine Disziplin, in unserem Fall der Kriminologie, solange sie den Anspruch erhebt, sämtliche Verhaltensdimensionen der »Kriminalfrage«, d.h. sämtliche Probleme der Gewalt und der Verletzung von Rechten sowie der damit zusammenhängenden sozialen Konflikte, in ihre eigene - fragmentarische - Grammatik einzubeziehen.

Die Kriminologie, wie auch jede andere juristische, soziologische, psychologische oder politische Disziplin, kann an sich nur isolierte Antworten geben. Somit favorisiert sie die Trennung der wissenschaftlichen Gemeinschaften und fördert die Institutionalisierung der verschiedenen Agenturen des Staates und der Zivilgesellschaft, die jeweils nur Fragmente der diversen sozialen Probleme behandeln - den Bedingungen ihrer je spezifischen Zeitlichkeit gemäß zu unterschiedlichen Zeitpunkten.

In bezug auf die bereits erwähnte Verhaltensdimension der Kriminalfrage kann sich epistemologisch und politisch ein neuer Diskurs nur legitimeren, sofern er sich quer zur traditionellen akademischen Teilung der wissenschaftlichen Arbeit artikuliert. Träger eines solchen Diskurses kann nur ein kollektives Subjekt sein, das sich durch die Teilnahme von Akteuren verschiedener wissenschaftlicher Gemeinschaften konstituiert. Dabei sollen nicht die traditionellen Regeln der »wissenschaftlichen « Kompetenz, sondern die Regeln des zivilen Engagements und des politischen Verlangens der Bürger sowie der lokalen und zentralen Institutionen maßgeblich sein. Nur ein solcher Diskurs und ein solches Subjekt sind in der Lage, ein gesellschaftliches Wissen zu produzieren, das sich sowohl vom demokratischen Modell der Interaktion zwischen Wissenschaft und Gesellschaft inspirieren läßt, als auch an den realen Bedürfnissen der Menschen orientiert.

Die Notwendigkeit eines solchen neuen Wissens wird vielleicht eindeutiger, wenn wir die aktuelle Situation des effizientistischen Strafrechts mit zwei anderen Konstruktionen vergleichen: einerseits mit den politischen und juristischen Dimensionen des »modernen« Gesellschaftsvertrags und andererseits mit den Aktionsprinzipien eines minimalen und garantistischen Strafrechts, das ein drittes Modell der Integration der wissenschaftlichen Diskurse, die sich mit der »kriminellen Frage« auseinandersetzen, darstellt.

\section{Strafrechtlicher Effizientismus und Wirksamkeit des Gesellschaftsvertrags}

Die Begriffe »Effizientismus« oder »Funktionalismus« bezeichnen Formen der Perversion des Strafrechts, die in unseren Tagen in Europa und in Amerika anzutreffen 
sind, in Staaten also, deren Verfassungen die Prinzipien des sozialen Rechtsstaats und des liberalen Strafrechts garantieren. Der Effizientismus ist eine neue Form des strafrechtlichen Notstands. Er kann im allgemeinen als Indiz einer Konfliktualität betrachtet werden, die mit einer doppelten Krise in Verbindung steht: Einerseits mit der wirtschaftlichen und sozialen Krise, die die Globalisierung und die neoliberalen Politiken erzeugen, und andererseits mit der Krise der Parteien und der anderer Repräsentationsinstanzen, die keine politische Mediation in den ökonomisch erzeugten Konflikten erlauben.

Das Strafrechtssystem profitiert von diesem vacuum potestatis und eignet sich die freigelassenen Räume an. Es hört auf, eine ultima ratio zu sein und wandelt sich zu einer prima ratio, zu einem zugleich repressiven und symbolischen Werkzeug, das für die Lösung sozialer Probleme benutzt wird. Sein repressiver Aspekt wird durch das quantitative und qualitative Wachstum der Strafen und vor allem der Raten der Einsperrung eindeutig, was einige Strafrechtskritiker dazu bringt, die Entwicklung seit Beginn der '80 Jahre als eine Entwicklung hin zur Bildung »neuer GULAGs, westlichen Stils « zu charakterisieren ${ }^{20}$. Die symbolischen Aspekte hängen vor allem mit den »Gesetzes-Manifesten« zusammen, mit denen die Politiker auf die Vorwürfe der öffentlichen Meinung, das Strafrecht sei »laxistisch«, reagieren - eine Reaktion, die ein »magisches Strafrecht« erzeugt, dessen Hauptfunktion der »Exorzismus« zu sein scheint $^{21}$.

Der Effizientismus versucht, die strafrechtliche Antwort härter zu gestalten und vorzuverlagern, indem er strafrechtliche und -prozessuale Garantien aufhebt; es handelt sich um Garantien, die zentrale Elemente des liberalen Strafrechts, der Verfassungen und der internationalen Konventionen bilden. Im Bereich der Strafgewalt führt aber jede Senkung des Niveaus der Garantien zur Zerstörung des Gleichgewichts zwischen materieller und prozeduraler Wahrheit und bringt uns zurück zu vormodernen Prozeßformen: Das Ermittlungsverfahren erzeugt die Beweise, die medialen Informationen kreieren den Kriminellen, das Verfahren bildet die Strafe ${ }^{22}$. Man steuert auf ein »totalitäres Modell der Kriminalpolitik« (Delmas-Marty, 1986, 190) zu, hin zu Formen einer »sanften Inquisition« (Padovani 1991), die mit dem liberalen und demokratischen System der verfassungsmäßigen Ordnung koexistieren.

Von dem Standpunkt der Systemtheorie aus betrachtet ${ }^{23}$, gibt uns der strafrechtliche Effizientismus ein klassisches Beispiel jenes Teufelskreises, den die Unwirksamkeit der strafrechtlichen Antwort in bezug auf bestimmte Probleme im Gang setzt. Der Effizientismus gibt keine kognitive, sondern eine normative Antwort. Von der Wirklichkeit »enttäuscht«, will er aus den Mißerfolgen keine Lehre ziehen und versucht die

20 Christie, 1994; zur Zukunft des Gefängisses s. die Diskussion in Scheerer, 1996.

21 Delmas-Marty, 1986, 44. Vgl. Hassemer, 1989; Albrecht, 1997, 128-131; zum »symbolischen Strafrecht«, s. Müller, 1993; Lehne, 1994.

22 Zur Kritik des Effizientismus im materiellen und prozeduralen Strafrecht s. u.a. Schumann, 1977; Baratta, Silbernagl, 1983; Baratta, 1989; Hassemer, 1990; Aponte, 1990; Albrecht, 1993; Frister, 1994; Moccia, 1997.

23 Ich verwende hier auf eigene Verantwortung Konzepte, die Luhmann in mehreren Arbeiten entwickelt hat (s. etwa Luhmann, 1993, 76-83, 133-134, 555-556). 
strafrechtliche Antwort wirksamer zu machen, indem er die Reaktion verhärtet, auch wenn dies zu Lasten des Funktionstüchtigkeit und der Legitimation der Strafjustiz geht $^{24}$.

Der Effizientismus indiziert jedoch nicht nur eine kognitive Unzulänglichkeit, der mit einigen guten Argumenten begegnet werden könnte. Er ist vor allem ein Verhaltensproblem: Er ist Bestandteil der aktuellen sozialen und politischen Krise und der Kommunikationsprozesse, die sie begleiten. Was auf dem Spiel steht, ist nicht nur die Form der Perzeption des Strafrechts durch die Juristen, die Politiker und die öffentliche Meinung, sondern vor allem die Form der Perzeption der sozialen Konflikte. Dies wird einsichtiger, wenn wir uns jene Deformationen näher betrachten, die diese falsche Perzeption charakterisieren. Es handelt sich um Ausdrücke einer Kriminalisierung der sozialen Konflikte bzw. um eine Lektüre dieser Konflikte nach dem Binom Verbrechen und Strafe.

Wir konstatieren zunächst eine »Ethisierung « oder »Verabsolutierung « der Konflikte. Ihr wirklicher Inhalt, der Strafanspruch, wird von einer ideologischen Polarisierung zwischen dem Guten und dem Bösen überlagert. Wir können somit eine Ausdehnung der moralischen Sphäre feststellen, die psychosoziale Projektionsmechanismen in Gang setzt. Somit werden Etikettierungen und »schweigende Mehrheiten « geschaffen; letztere interagieren mit den moralistischen Kreuzzügen und den »Lawand-Order«-Kampagnen, die den politischen Konsens und die Legitimation der Macht beeinflussen.

Zweitens erfolgt eine »Entkontextualisierung « und eine »Entpolitisierung « der Konflikte. Durch eine stark vereinfachende Lektüre der Konflikte, wird der strafrechtliche Weg als Allheilmittel für das Verständnis und die Bewältigung von besonders komplexen Phänomenen, wie der Mafia oder großer krimineller Organisationen, des Terrorismus, der Korruption in der Verwaltung, der kriminellen Abweichungen des Militärs oder anderer repressiver Staatsorgane sowie der Zerstörung der Umwelt, dargestellt.

Drittens kann man eine »Technisierung « der Konflikte feststellen, die auf der Basis der bereits erwähnten komplexen Phänomene steht. Dies ist im Grunde genommen eine Konsequenz der Entpolitisierung dieser Konflikte, die jedoch nur partiell und auf der Ebene der Symptome gelöst werden können, wenn sie der ausschließlichen Kompetenz der kriminologischen Disziplinen und der Organe der Strafjustiz überlassen werden, wenn sie also dem Bereich der Politik und der öffentlichen Diskussion entzogen werden. Die reiche Geschichte der staatlichen »Antworten « auf die italienische Mafia sind ein besonders lehrreiches Beispiel dafür ${ }^{25}$.

Ebenso bemerkenswert ist die Tatsache, daß die Kriminalisierung dieser Konflikte sehr oft zu ihrer Entpönalisierung führt. Die Vermehrung der Straftaten im Rahmen des Effizientismus vermehrt auch die Fälle der Straflosigkeit, die selbst im Rahmen des »normalen « Strafrechts einen besonders großen prozentualen Anteil an den prinzipiell strafbaren Taten ausmachen, wie die Forschung zum Dunkelfeld und zur Selekti-

$24 \mathrm{Zu}$ ähnlichen Reaktionen in bezug auf die Krise des Gefägnisses s. Foucault (1975, 273274).

25 Baratta 1994a m. w. Hinw. 
vität des Strafrechtssystems zeigt. Der Effizientismus vergrößert über jedes Ausmaß die Aktionsprogramme des Strafrechts, ohne daß es von einem entsprechenden Wachstum der Verfolgungsraten begleitet werden kann - was zu einer vollkommenen Militarisierung der Gesellschaft führen würde ${ }^{26}$. Das Ergebnis ist ein ständiger Ausbau selektiver Mechanismen, wobei die traditionellen Variablen der Selektivität unverändert bleiben. Das praktische Ergebnis des effizientistischen Strafrechts ist, daß die Gefängnispopulation hauptsächlich aus »schwachen« Subjekten zusammengesetzt ist. In einigen europäischen Ländern, die effizientistische Programme anwenden, besteht fast die Hälfte der Gefangenen aus Drogensüchtigen und sog. »Drittausländern « ${ }^{27}$.

Unter dem Vorwand der Erhöhung der Effizienz des Strafrechtssystems ist der Effizientismus immer bereit, Zugeständnisse zu machen bei der Respektierung der Grenzen der Strafgewalt, die das Hauptmerkmal seiner Normalität ausmachen. Somit steht er im Widerspruch mit den konstitutiven Merkmalen des Gesellschaftsvertrags und des Systems der Grundrechte. Darüber hinaus hat jede Umwälzung der Normalität des Strafrechts schwerwiegende Konsequenzen für seine Effizienz, auch wenn sie zunächst von den Ergebnissen einer effizientistischen Verfolgungspolitik kompensiert werden. Und dies weil es nicht nur unmöglich ist, zu beweisen, daß dieselben Ergebnisse nicht unter Respektierung der Verfassungsnormen im Bereich des Strafrechts erzielt werden könnten, sondern auch weil in dem allgemeinen Kalkül der Effizienz des Rechtssystems eines Rechtsstaats die Verstöße seitens der Staatsorgane einen negativen Einfluß auf das Vertrauen der Bürger und auf den sozialen Konsens haben, also auf Elemente, die die Effizienz des Sozialpakts bestimmen.

Die Gewalt der Konflikte »barbarisiert« das Strafrecht, und umgekehrt »barbarisiert« die Gewalt der Bestrafung außerhalb der Regeln eines sozialen Rechtsstaats die Konflikte. Das Programm eines minimalen Strafrechts (Baratta, 1988) oder eines Strafrechts in den Grenzen der Verfassung (Bricola, 1973 und 1984) ist nicht nur ein Zukunftsentwurf, d. h. eine Herausforderung des sozialen Friedens und der Konfliktlösung. Es ist auch ein rechtlicher Ausdruck der »Rechtfertigungsgründe « des modernen Staates, die oft mit den Termini Gesellschaftsvertrag und Versprechen der Moderne umschrieben werden.

Der Gesellschaftsvertrag, der dem modernen Recht und den demokratischen Verfassungen entspricht, ist mit dem Versuch verbunden, den Krieg zu bändigen, die politischen und sozialen Konflikte zu zivilisieren und sie institutionalisierten Regeln zu unterwerfen $^{28}$. In diesem Rahmen bildet die Sicherheit der Bürger das zentrale Versprechen des Staates (Lehne, 1996, 309). Die Geltungs- und Wirksamkeitsbedingung dieses Vertrags ist in der Tat die Beseitigung der Gewalt durch die Zentralisierung der legitimen Macht/Gewalt in den Apparaten eines »überparteiischen « Staates. Das geschichtliche Ergebnis dieses Versuchs führte zur sog. Krise der Moderne und zur entsprechenden Kritik postmoderner Autoren: das moderne Recht, das die Gewalt auf

26 S. etwa Hassemer, 1989, 558-559; Müller, 1993, 86-87.

27 Sim, Ruggiero, Ryan, 1995, 10-11; Ruggiero, 1995, 48-49; Bergalli, 1995, 157; Tournier, 1996, 133, 137.

28 Zu diesem Interpretationsschema s. ausführlich Baratta, Giannoulis, 1996, 253-266. 
institutionellem Wege zu beseitigen versucht, hat im Ergebnis die Präsenz der Gewalt verdrängt; sie hat die schwächeren Subjekte vom Vertrag faktisch ausgeschlossen und die Ungleichheit und die strukturelle Gewalt in der Gesellschaft auf juristischer Ebene unsichtbar gemacht ${ }^{29}$.

Symptomatisch dafür ist der Fall der »öffentlichen Strafe«. Die Ideologie der Aufklärung und die Gesetzgebung der Verfassungsstaaten versuchte, die Grausamkeit der vormodernen Strafen zu vermindern, indem sie die Strafe als ein Medikament verordneten, das zwar bitter, aber gegen die Gewalt wirksam war ${ }^{30}$. Die Strafgewalt hat sich eher als ein Gift erwiesen, das der staatlichen und sozialen Gewalt Nahrung gibt. Die Wechselbeziehungen zwischen gesellschaftlichen Konflikten und Kriminalisierungsprozessen, zwischen Ungleichheit und Repression, zwischen struktureller und strafrechtlicher Gewalt, bilden eine Konstante moderner Gesellschaften.

Wenn die Konflikte die Form eines Bürgerkriegs annehmen, dann beobachtet man im allgemeinen eine reziproke Beeinflussung des aggressiven Denkens und Handelns und der Form der Bestrafung. Dies trifft nicht nur bei den informellen Kriminalisierungsprozessen $\mathrm{zu}$, sondern auch im Rahmen des insitutionalisierten »parallelen « Strafsystems, das in quasinatürlicher Weise die bewaffneten Konflikte begleitet. Die Ordnungskräfte und das Strafrechtssystem werden kriegerisch; gleichzeitig erweitert sich der Aktionsradius der Bestrafung und involviert die Haltungen und die Praktiken der militärischen und paramilitärischen Organisationen, der bewaffneten Gruppen und der terroristischen oder kriminellen Organisationen.

Falls die Konflikte sich entschärfen und ihre Streitobjekte sich beschränken, dann verschwindet tendenziell auch die reziproke Konditionierung des Kriegs und der Strafe bzw. der Waffengewalt und der Strafgewalt. Die notwendige Bedingung einer Normalisierung des Strafrechtssystems ist, daß der Staat eine wirksame Kontrolle über die parallelen Systeme ausübt, so daß die Fortsetzung des Krieges verhindert werden kann und den sozialen Konflikten ihre kriegerische Schärfe genommen wird. Die Normalität des Strafrechtssystems erscheint dabei als Konsequenz der ideellen Geltung und der tatsächlichen Wirksamkeit des Gesellschaftsvertrags und der Verfassung ${ }^{31}$.

Was die Respektierung des Gesellschaftsvertrags betrifft, bildet der Frieden eine notwendige nicht aber eine hinreichende Bedingung. Die hinreichenden Bedingungen sind in der Wirksamkeit der Normen zu suchen, die die Organisation und die Trennung der Staatsgewalten bilden und die Grundrechte aller Bürger/Menschen ${ }^{32}$ garantieren und für den modernen Staat, wie Art. 16 der französischen Erklärung der Menschen- und Grundrechte von 1789 sagt, wesensbestimmend sind (Schmitt, 1989, 125-220).

29 Galtung, 1975, 7-36; in bezug auf das Strafrechtsystem s. Baratta, 1993a.

30 Für eine Analyse der Beziehungen zwischen institutioneller und »wilder « Gewalt unter Verwendung der Metapher des pharmakon (griechisch: Gift und Heilmittel) vgl. Resta, 1992.

31 Vgl. Orozco Abad, Aponte, 1992, 219-232 und passim.

32 Die Identität dieser Begriffe ist keine institutionelle Gegebenheit, sondern eine politische Forderung und eine Bedingung der effektiven Demokratie; dazu s. Balibar, 1989, 4-6, 11-15. 
Die konkrete Utopie einer Allianz aller Opfer der Moderne und die neue Formulierung des Gesellschaftsvertrags in einer Perspektive der Inklusion der Ausgeschlossenen zeigt den politischen Weg für ein Projekt auf planetarischer Ebene, das zu Formen einer effektiven »menschlichen Entwicklung « führen könnte ${ }^{33}$, in der die Beziehungen zwischen Bedürfnissen, Fähigkeiten und Grundrechten ein höheres Stadium erreichen würden.

Dieser Weg der friedlichen politischen Kämpfe führt auch über eine neue Interpretation und eine dynamische Anwendung der Verfassung der sozialen Rechtsstaaten ${ }^{34}$ sowie über eine Politik des sozialen Fortschritts sowie des umfassenden Schutzes der Grundrechte, die die Verfassungen, mindestens in der Form allgemeiner Prinzipien staatlichen Handelns, garantieren. Wenn wir diesen Weg nicht einschreiten, dann wird die Erfüllung der Bedingungen eines normalen Strafrechts unmöglich, auch dort, wo seine notwendige Bedingung, der Frieden, bereits realisiert ist.

\section{Von der Kriminalpolitik zur Politik des Schutzes der Grundrechte}

Wir haben bereits gesehen, daß die Kriminologie in ihrer Dimension des Verhaltens keine Zukunft hat. Wie verhält es sich nun mit der Kriminalpolitik, die größtenteils ein praktischer Ausdruck kriminologischer Theoreme bezüglich des Verhaltens ist? Um diese Frage angemessen zu behandeln, müssen wir zuerst unterstreichen, daß Hauptzweck der Kriminalpolitik die Herausarbeitung von Aktionsprogrammen zur Bewältigung folgender Sachverhalte ist:

- problematische Situationen der Verletzung von Grundrechten, die mit dem Verhalten von Individuen verbunden sind;

- Prozesse der Kriminalisierung;

- persönliche und soziale Konsequenzen der Verletzung von Grundrechten sowie der Kriminalisierungsprozesse.

Dies entspricht einer dreifachen Aufgabe, die die Kriminalpolitik mit Hilfe der kritischen Richtungen der Kriminologie zu bewältigen hat. Es ist allerdings offensichtlich, daß die Kriminalpolitik auch das Problem der Heterogenität und des unbestimmten Charakters des Gegenstands der Kriminologie zu lösen hat: ihr fehlt nämlich, im Vergleich zu den - artifiziellen und kontingenten - Abgrenzungen der legalen Definitionen, die Möglichkeit, eine Grenze zu ziehen.

Was die Instrumente der Kontrolle der problematischen Situationen betrifft, so beobachten wir in den letzten Jahren eine bedeutende Erweiterung des Reaktionsarse-

33 Dieser Begriff wird von Organen der UNO benutzt. Die drei grundsätzlichen Dimensionen der »menschlichen Entwicklung « sind die Gesundheit und die Lebenserwartung, die Erziehung und der Zugang zu materiellen Ressourcen, die einen zufriedenstellenden Lebensstandard erlauben. Die Forscher des Entwicklungsprogramms der UNO analysieren in ihren jährlichen Berichten die Entwicklung mehrerer Indikatoren, die eine Quantifizierung der menschlichen Entwicklung auf Weltebene erlauben. Zur Definition des Konzeptes s. UNDP, Human Development Report 1990, New York, 9-16; zum »Recht auf Entwicklung « s. Denninger, 1990, 221; Tetzlaff, 1993; Kößler, 1998.

34 Denninger, 1990, 27 und 226; Canotilho, 1982, 349 und passim. 
nals selbst im Rahmen des Strafrechtssystems. Der immer intensivere Rekurs auf die sog. alternativen Strafen wird mit dem Versuch verbunden, das Sanktionssystem neu zu definieren und die zentrale Position der Freiheitsstrafe im Rahmen des »modernen « Strafrechts in Frage zu stellen ${ }^{35}$. Das Feld, das dadurch für die politische Kreativität eröffnet wird, erweitert sich, wenn wir den Bereich der alternativen Strafen verlassen und über Alternativen zur Strafe nachdenken, uns also Gedanken über eine nicht strafrechtliche Prävention machen. Dadurch wird der Übergang von der alternativen Kriminalpolitik zu den Alternativen zur Kriminalpolitik möglich.

Die Konstruktion des Gegenstands und der Instrumente der Kontrolle hat eine Identitäts- und Kompetenzkrise der Kriminalpolitik hervorgerufen, die viel stärker als diejenige der Kriminologie ist. Wenn die externe Interdisziplinarität für die Kriminologie erforderlich ist, ist sie um so mehr für die Kontrolle der sozialen Probleme vonnöten, nicht nur um das »Objekt « der Intervention besser zu definieren, sondern auch um die Instrumente der Kontrolle durch die Begegnung verschiedener wissenschaftlicher Gemeinschaften angemessen zu bestimmen. Ein wertender Austausch der Erfahrungen verschiedener Instanzen des Staates, der Selbstverwaltungsorgane und der Vertreter der Zivilgesellschaft könnte zur Herausbildung eines kollektiven Subjekts führen, das - gemäß der Spezifizität jedes Problems und der Pluralität der Strukturen, die die problematischen Situationen verwalten und die Instrumente der Kontrolle definierenadäquate Ressourcen und Kompetenzen zusammentragen könnte. Dieser Weg sollte »vom Einfachen zum Komplexen, vom Einzigen zum Multiplen« führen (DelmasMarty, 1986, 214).

Hier gibt es zwei gleichermaßen unbefriedigende Optionen. Entweder wird der Kriminalpolitik ein heterogener und kaum bestimmter Tätigkeitsbereich zugewiesen, oder ihre Spezifizität wird in der Erzeugung, der Anwendung und der Reform der Regeln des Strafrechtssystems gesucht. Im letzten Fall würde die Kriminalpolitik zwar immer noch ein sehr weites Aktionsfeld erhalten, sie würde sich jedoch als Politik des Strafrechts beschränken. Hier wird nicht bezweifelt, daß das Strafrecht eine notwendige Bedingung der Kontrolle und der Beschränkung der Strafgewalt bildet ${ }^{36}$; man sollte diesen Status aber nicht zu einer hinreichenden Bedingung erheben. Man sollte versuchen, aus der heutigen Sackgasse einen Ausweg zu finden, der sowohl den Teufelskreis des Effizientismus, als auch die Kriminalisierung der Sozialpolitik vermeidet.

Eine gemeinsame Anstrengung juristischer Erfindungsgabe und politischer Phantasie könnte zu einer Emanzipation der politischen Tätigkeit von der »Strafkultur« führen. Hier ist eine neue Lektüre der menschlichen Bedürfnisse und der Risikositua-

35 Foucault $(1975,119,234)$ analysiert die »Kolonisierung « des Strafrechtssystems von dem Gefängnis in einem Werk, das der Interpretation der sozialen und politischen Evidenz des Gefängnisses gewidmet ist. Delmas-Marty, 1986, 70 spricht von der »Äquivalenz Strafe = Gefängnis «, die in den legislativen und theoretischen Entwicklungen der letzten Jahrzehnte in Frage gestellt wird; dazu vgl. Eusebi, 1990.

36 Aus diesem Grund habe ich die Forderung der Kohärenz zwischen den verfassungsrechtlichen Prinzipien der Strafgewalt und der Realität der Repressionsapparate als eine historische Herausforderung für das moderne Strafrecht bezeichnet (Baratta, 1993, 415; 1988, $518 \mathrm{ff}$.). 
tionen in der Perspektive des Verfassungssystems notwendig; daraus sollte nicht nur der Entwurf eines Strafrechts in den Grenzen der Verfassung entstehen, sondern auch eine neue Definition der Politik im Lichte des Verfassungsprojekts als einer Politik der Grundrechte.

Zweck dieser Arbeit ist die Gewährleistung der Autonomie und der Zentralität der Grundrechte im Rahmen der Kriminalpolitik im weiten Sinne. Eine Politik der sozialen Gerechtigkeit und der Gleichheit kann nämlich nur positive Auswirkungen auf die Kontrolle der Kriminalität und der Prozesse der Kriminalisierung haben. Die Politik des Strafrechts ist, was von der Kriminalpolitik übrigbleibt, wenn aus ihr alle Operationen entfernt werden, die höchstens indirekt die Kontrolle der Kriminalität und der Kriminalisierungsprozesse beeinflussen, während sie sich auf den Schutz der Grundrechte unmittelbar und negativ auswirken. Wenn wir die Kriminalpolitik nicht ausdehnen wollen, so daß sie faktisch die Stelle der Sozialpolitik einnimmt, dann soll man sie auf eine Politik des Strafrechts beschränken, die ihrerseits einen integrierenden - wenn auch subsidiären und residuellen - Bestandteil einer umfassenden Politik des Grundrechtsschutzes bildet.

\section{Kriminalpolitik und Verfassung als Projekte. Zur Theorie und Praxis eines »positi- ven Garantismus«}

Das moderne Strafrecht wurde immer als Gründung einer neuen Form der Bestrafung betrachtet. Die Erfahrung von zwei Jahrhunderten und die heutige Entlegitimierung der Bestrafungssysteme und des Rechts, das diese Systeme regulieren sollte (Zaffaroni 1989), erlaubt uns eine andere Interpretation zu formulieren: das liberale Strafrecht war vor allem ein Versuch der Moderne, die Strafen zu regulieren und in Schranken zu halten: die moderne Strafe ist aber an sich eine vormoderne Reaktionsform, die nur die Formen des Leides ändert ${ }^{37}$. Die Bestrebung des modernen Rechts, die Strafgewalt zu regulieren $^{38}$, gibt letzterer höchstens eine Legitimierung die genauso minimal ist, wie diejenige, die das internationale und humanitäre Recht dem Krieg gibt, indem es seine Gewalt zu kontrollieren versucht.

Das Strafrecht in den Grenzen der Verfassung befindet sich in derselben Situation, die das Strafrecht der Aufklärung seinerzeit erlebt hatte. Damit ersterem nicht dasselbe Schicksal ereilt, wie dem zweiten, welches größtenteils im Kopf seiner Theoretiker verblieb, ist es vonnöten, daß es eine ausgeprägte und authentische politische Dimension erlangt. Das ist aber nur dadurch zu erreichen, wenn es sich eine integrale Politik des Grundrechtsschutzes einverleibt.

In den 80er Jahren hatte ich vorgeschlagen, im Rahmen einer Strategie des minimalen Strafrecht eine epoché, d. h. eine methodische Suspendierung der Begriffe des

37 Christie, 1986; Baratta, 1998.

$38 \mathrm{Zu}$ einer fundierten und umfassenden rechtssoziologischen Analyse und Kritik des Strafrechts - das sich weiterhin von seinen erklärten rechtsstaatlichen Prämissen entfernt - als einer derjenigen Institutionen, die insgesamt und auf eine spezifische Weise die gesellschaftliche Differenzierung reproduzieren, s. die grundlegende Arbeit von Smaus 1998. 
Delikts und der Strafe, vorzunehmen ${ }^{39}$. Meine Absicht war sicherlich nicht, das Bestehen sozial negativer Situationen zu leugnen noch übersah ich die Wirklichkeit des Strafsystems. Die epoché war vielmehr eine Einladung zu mehr Empfindsamkeit, um diese Situationen durch Verwendung alternativer Konzepte zu rekonstruieren, so daß andere Eingriffsformen möglich werden. Andere Disziplinen und Kontrollinstanzen könnten einberufen werden, um diese Probleme angemessener zu behandeln. Dieser Vorschlag war jedoch nicht von einer Reflexion der epistemologischen und praktischen Probleme begleitet, die eine Rekonstruktion der zu kontrollierenden Situationen stellt, d. h. von Problemen, die aus der Konvergenz unterschiedlicher methodischer Ansätze und Operationen entstehen.

Wenn dabei von einem Perspektivenwechsel die Rede sein kann, dann betrifft dieser nur die Aufgabe der Idee einer Trennung der verschiedenen Ansätze und Kontrollinstanzen. Dabei ist ein Koordinationsprinzip unentbehrlich, welches die Koexistenz und die Zusammenarbeit erlaubt und die reziproke Lernfähigkeit fordert bezüglich der Bewertung und der Behandlung der problematischen Situationen.

Die Disziplinen und die Instanzen des Strafrechtssystems sollten nicht von vornherein von dieser $»$ Werkstätte« ausgeschlossen sein. Ihre Einbeziehung ist jedoch weder »natürlich« noch immer notwendig. Das Subsidiaritätsprinzip bezüglich eines Strafeingriffs ist auch als Regel interdisziplinäre Methodik anzuwenden. Im Fall eines Zweifels oder einer Kontroverse fällt die Beweislast auf diejenigen, die die Notwendigkeit der strafrechtlichen Komponente behaupten.

So zeichnet sich ein drittes Modell der Integration der wissenschaftlichen Diskurse ab, welches aus der Regel der Interdisziplinarität und der Pluralität der Instanzen seinen Antrieb erhält. Dieses Modell berücksichtigt den hohen Grad der Differenzierung, den die Instrumente des Strafrechts erreichen können (Delmas-Marty, 1986, Lüderssen, 1995) und erlaubt, Strategien zum Schutze der Grundrechte zu entwerfen, welche die strafrechtlichen Eingriffe mit präventiven und reaktiven »Antworten«, die aus anderen Instanzen kommen und sich aus anderen Wissensquellen speisen, koordinieren. Die Idee der Schaffung eines kollektiven Subjekts, im Sinne eines offenen und beweglichen Zusammenschlusses von Denk- und Handlungskräften, zeigt die Notwendigkeit, interdisziplinäre Werkstätten zu öffnen, die dem Problem der Sicherheit der Rechte gewidmet sein werden.

Im Rahmen einer umfassenden Politik des Grundrechtsschutzes ist das Strafrecht, wie bereits erwähnt, ein Residuum und führt zu einem strafenden Eingriff, wenn besonders schwerwiegende Verletzungen der Grundrechte oder ein unvermeidbares soziales Verlangen keine andere Reaktionsmöglichkeit erlauben. Als Strafrecht in den Grenzen der Verfassung impliziert das minimale Strafrecht einen ständigen Versuch, die Mechanismen der Kriminalisierung und die Änderungen in der Gesetzgebung, der Rechtsprechung und dem Vollzug zu kontrollieren ${ }^{40}$.

39 Baratta, 1988, 536 f.; 1993, 20-24.

40 Eine solche Kontrolle impliziert nicht nur die Verminderung der Gewalt und des Aktionsradius der repressiven Apparate (wie die Forschungen zum Unvermögen des Strafrechtssystems, seine erklärten Funktionen angemessen zu erfüllen, zeigen: s. die Übersicht bei 
Es handelt sich also vor allem um den Versuch, die Verfassungsprinzipien im strafrechtlichen Bereich anzuwenden und die Ergebnisse der Forschung bezüglich der Funktionen des Strafrechtssystems und der geeigneten Strategien zum Schutz der Grundrechte anzuwenden. Gleichzeitig handelt es sich um einen Versuch, die lokalen Erfahrungen und Programme auf zentrale Ebene zu projizieren zum Zweck einer autonomen Artikulation der jeweiligen Konflikte und Bedürfnisse (Baratta, 1988, 538 f.).

Die Perspektive des Strafrechts in den Grenzen der Verfassung gemäß den Prinzipien einer umfassenden Politik zum Schutze der Grundrechte zu erweitern heißt, unter anderem, den Garantismus nicht nur als eine Schranke des Bestrafungssystems oder nur als einen Ausdruck der Rechte der Bürger zum Schutze vor der Staatsgewalt zu definieren (negativer Garantismus). Erforderlich ist vor allem ein positiver Garantismus ${ }^{41}$ als Antwort des Staates auf Bedürfnisse zur Sicherung aller Leistungsrechte und nicht nur zum Schutze ihres kleinen Teils, den das Recht zur Schutzleistung vor dem deliktiven Verhalten bestimmter Personen ausmacht.

Ein Teil der heutigen Unsicherheit der Bürger ist tatsächlich auf Rechtsverletzungen zurückzuführen, die mit dem deliktiven Verhalten bestimmter Personen zusammenhängt. Es reicht jedoch bei weitem nicht aus, vor der Kriminalität (und vor den Kriminalisierungsprozessen) zu schützen. Die Sicherheit entspricht dem Bedürfnis, sicher zu sein und sich sicher zu fühlen bei der Ausübung aller Rechte: Recht auf Leben, Freiheit, Entwicklung der Persönlichkeit und der Fähigkeiten, auf Meinungsäußerung und Kommunikation, auf einen hohen Standard der Lebensqualität, sowie auf effektive Kontrolle und Beeinflussung der Bedingungen, von denen die Existenz eines jeden abhängt ${ }^{42}$.

Baratta, 1981; 1982; 1993, 407 ff.), sondern auch eine Transformation dieser Apparate durch eine neue Bestimmung ihrer Rollen, Prioritäten sowie der Akteure der Prävention und der Repression. So müssen wir die Frage stellen, ob es möglich ist, dem Gefängnis neue Funktionen zuzuweisen, also Funktionen, die sich von den aktuellen grundsätzlich unterscheiden. Eine solche Umwandlung setzt den Willen voraus, die Wege einer symbolischen und wirklichen Öffnung des Gefängnisses zur Gesellschaft zu experimentieren (Baratta, 1982, 206) und vor allem die Insassen als Grundrechtsträger und nicht als »Behandlungsobjekte« eines von oben geleiteten »Strafvollzugs« zu betrachten. In einer solchen Perspektive wäre das Gefängnis kein Ort der »Vorbereitung « einer Eingliederung in die Gesellschaft mehr, sondern einer der vielen Bereiche der Ausübung der Bürgerrechte der Insassen, die in das soziale Handeln (immer) schon einbezogen sind; vgl. Baratta 1994 und das Heft »Art et pensée en prison« der Zeitschrift Lignes, Nr. 27, 1996, insbesondere die dortige Analyse von Balibar, 1996.

41 Zum Garantismus s. das grundlegende Werk von Ferrajoli (1990, 329-340 und passim). Ferrajoli definiert das Strafrecht normativ als das »Recht der Schwächeren«. Somit wird eine Variante des positiven Garantismus mit strafrechtlicher Dominante eingeführt, die dem Strafrecht ein weites Aktionsfeld anerkennt, da es imstande wäre, die Grundrechte zu schützen. Meiner Meinung nach soll dagegen ein positiver Garantismus mit einer nichtstrafrechtlichen (und allgemeiner mit einer nicht staatlich-juristischen) Dominante verteidigt werden, aufgrund der strukturellen Beobachtung, daß das Strafrecht immer die Interessen der politisch und gesellschaftlich »stärkeren« Gruppen schützt; vgl. Baratta 1996.

42 Denninger, 1990, 257-259; zum »empowerment« s. Friedmann, 1992; Frankenberg 1994. 
Die Beziehung zwischen dem negativen und dem positiven Garantismus ist analog zur Beziehung zwischen einer Politik des Strafrechts und einer umfassenden Politik des Grundrechtsschutzes. Das Ganze benötigt all seine Bestandteile; kein Teil kann jedoch ohne das Ganze überleben.

\section{Bibliographie}

ALBRECHT P.A., Erosionen des rechtsstaatlichen Strafrechts, Kritische Vierteljahresschrift für Gesetzgebung und Rechtswissenschaft, 1993, 2, 163-182

ALBRECHT P.A., La politique criminelle dans l'Etat de prévention, Déviance et société, 2, 1997, 123-135

ALEXY R., Theorie der Grundrechte, Frankfurt/M., Suhrkamp, 1986

APONTE A.D., Cómo matar a la justicia en la tarea de defenderla: »Estatuto para la defensa de la justicia «, Análisis politico, 11, 1990, 77-90

BALIBAR E., La proposition de l'égaliberté, Paris, Les Conférences du Perroquet, no 22, 1989

BALIBAR E., Murs contre murs (impressions de Saint-Maur), Lignes, 27, 1996, 63-77

BARATTA A., Strafrechtsdogmatik und Kriminologie. Zur Vergangenheit und Zukunft des Modells einer gesamten Strafrechtswissenschaft, ZStW, 92, 1980, 107-142

BARATTA A., Remarques sur la fonction idéologique du pénitencier dans la reproduction de l'inégalité sociale, Déviance et société, 2, 1981, 113-131

BARATTA A., Criminologia critica e critica del diritto penale, Bologna, Il Mulino, 1982

BARATTA A., Prinzipien des minimalen Strafrechts. Eine Theorie der Menschenrechte als Schutzobjekte und Grenze des Strafrechts, in: G. Kaiser/H. Kury/H.-J. Albrecht (Hg.), Kriminologische Forschung in den 80er Jahren, Freiburg i. Br. 1988, 513-542

BARATTA A., Il nuovo rito penale: la posta in gioco, in Il nuovo rito penale, fascicolo monografico de »la difesa penale«, Latina, Bucalo, 1989, 231-251

BARATTA A., Jenseits der Strafe. Rechtsgüterschutz in der Risikogesellschaft, in: F. Haft et al., Festschrift für Arthur Kaufmann, Heidelberg, Müller, 1993, 393-422

BARATTA A., Die Menschenrechte zwischen struktureller Gewalt und strafrechtlicher Strafe, in MARTINEK M., SCHMIDT J., WADLE E., Ed., Festschrift für Günther Jahr, Tübingen, Mohr, 1993a, 9-24

BARATTA A., I nuovi orizzonti della prevenzione, Sicurezza e territorrio, 2, 1993b, 9-14

BARATTA A., Reintegrazione sociale. Ridefinizione del concetto ed elementi di operazionalizzazione, Dei Delitti e delle Pene, 3, 1994, 137-150

BARATTA A, Mafia e Stato. Alcune riflessioni metodologiche sulla costruzione del problema e la progettazione politica, in G. FIANDACA, S. COSTANTINO, Ed., La mafia, le mafie, Roma-Bari, Laterza, 1994a, 95-117

BARATTA A., Prefazione, in MOCCIA S., La perenne emergenza, Napoli, ESI, 1996, XIXXVI

BARATTA A., Das Theater des Rechts und die Dramaturgie des Lebens. Zur Zurechnung von Verantwortlichkeit im Strafprozeß, in: H. Jung (Hg.), Festschrift Müller-Dietz, BadenBaden 1998, 133-159

BARATTA A., GIANNOULIS Ch., Vom Europarecht zum Europa der Rechte, Kritische Vierteljahresschrift für Gesetzgebung und Rechtswissenschaft, 3, 1996, 237-266

BARATTA A., SILBERNAGL M., La legislazione dell'emergenza e la cultura giuridica garantista nel processo penale, Dei delitti e delle pene, 3, 1983, 543-580

BARATTA A., SILBERNAGL M., Neue Legitimationsstrategien des Strafrechts und ihre Kritik als Realitätskritik, Kriminologisches Journal, 1, 1988, 32-49

BERGALLI R., The Spanish Attempt to Build a Democratic Criminal Justice System, in RUGGIERO V., RYAN M., SIM J., Ed., Western European Penal Systems. A Critical Anatomy, London, Sage, 1995, 149-168 
BRICOLA F., Teoria generale del reato, Novissimo Digesto italiano, vol. XIX, Torino, Utet, 1973, 8-93

BRICOLA F., Tecniche di tutela penale e tecniche alternative di tutela, in DE ACUTIS M., PALOMBARINI G., Ed., Funzioni e limiti del diritto penale, Padova, Cedam, 1984, 3-84

CANOTILHO J.J., Constituição dirigente e vinculação do legislador, Coimbra, Coimbra editora, 1982

CHAPMAN D., Sociology and the Stereotype of the Criminal, London, Tavistock, 1968

CHEVALIER G., L'intérêt central pour le local. Analyse des politiques socio-préventives en France entre 1981 et 1986, Déviance et société, 3, 1988, 237-267

CHRISTIE N., Grenzen des Leids, Bielefeld, AJZ, 1986

CHRISTIE N., Crime Control As Industry: Towards Gulags Western Style, London, Routledge, 1994

CREAZZO G., Le politiche di »nuova« prevenzione: lo stato dell'arte, Quaderni di Città sicure, 4, 1996, 13-28

DELMAS-MARTY M., Modèles et mouvements de la politique criminelle, Paris, Economica, 1983

DELMAS-MARTY M., Le flou du droit. Du Code Pénal aux Droits de l'Homme, Paris, P.U.F., 1986

DENNINGER E., Der gebändigte Leviathan, Baden-Baden, Nomos, 1990

DUPREZ D., Le modèle francais de prévention de la délinquance: la recherche d'un second souffle, in HEBBERECHT P., SACK F., Ed., La prévention de la délinquance en Europe. Nouvelles stratégies, Paris, L'Harmattan, 1997, 61-82

DWORKIN, R., Bürgerrechte ernstgenommen, Frankfurt./M., Suhrkamp, 1990

EUSEBI L., La pena »in crisi«. Il recente dibattito sulla funzione della pena, Brescia, Morcelliana 1990

FERRAJOLI L., Diritto e ragione. Teoria del garantismo penale, Roma, Laterza, 1990

FOUCAULT M., Surveiller et punir, Paris, Gallimard, 1975

FRANKENBERG, G., Solidarität in einer »Gesellschaft der Individuen «? Stichworte zur Zivilisierung des Sozialstaats, in FRANKENBERG, G. (Hg.), Aud der Suche nach der gerechten Gesellschaft, Frankfurt/M., Fischer, 1994, 210-223

FRIEDMANN J., Empowerment. The Politics of Alternative Development, Cambridge, Blackwell, 1992

FRISTER H., Beschleunigung der Hauptverhandlung durch Einschränkung von Verteidigungsrechten?, Strafverteidiger, 8, 1994, 445-454

FROMMEL M., Umrisse einer liberal-rechtsstaatlichen Normverdeutlichung durch Strafrecht, in ALBRECHT P.-A., Ed., Festschrift für Horst Schüler-Springorum, Köln, Heymann, 1993, 257-276

GALTUNG J., Strukturelle Gewalt, Beiträge zur Friedens- und Konfliktforschung, Reinbek b. Hamburg, Rowohlt, 1975

GARCIA MENDEZ E., Recht und Autoritarismus in Lateinamerika, Frankfurt/M., Vervuert, 1985

GASSIN R, Criminologie, Paris, Dalloz, 1988

GRÄFRATH B., HUBER R., UHLEMANN B., Einheit, Interdisziplinarität, Komplementarität. Orientierungsprobleme der Wissenschaft heute, Berlin, De Gruyter, 1991

HANNOVER H., Kollaboration mit der Justiz als Kriterium der Freund-Feind Unterscheidung, Kritische Justiz, 4, 1989, 394-408

HASSEMER W., Symbolisches Strafrecht und Rechtsgüterschutz, Neue Zeitschrift für Strafrecht, 1989, 12, 553-559

HASSEMER W., Das Schicksal der Bürgerrechte im »effizienten« Strafrecht, Strafverteidiger, 1990, 7, 328-331

HEBBERECHT P., SACK F., Ed., La prévention de la délinquance en Europe. Nouvelles stratégies, Paris, L'Harmattan, 1997

HESS H., Kriminalität als Alltagsmythos, Kriminologisches Journal, Beiheft 1, 1986, 24-44 
HESS H., SCHEERER S., Was ist Kriminalität? Skizze einer konstruktivistischen Kriminalitätstheorie, Kriminologisches Journal, 2, 1997, 83-155

JUNG H., Das Opferschutzgesetz, JuS, 2, 1987, 157-160

KAISER G., Kriminologie, Müller, Heidelberg, 1985

KÖSSLER, R., Recht auf Entwicklung, Widerspruch, 1998, 25-33

KRAUSS D., La vittima del reato nel processo penale, Dei delitti e delle pene, 2, 1983, 283-298

LAZERGES C., La politique criminelle, Paris, P.U.F., 1987

LEHNE W., Symbolische Politik mit dem Strafrecht. Versuch einer Reinterpretation des Diskurses um symbolisches Strafrecht, Kriminologisches Journal, 3, 1994, 210-224

LEHNE W., Präventionsräte, Stadtteilforen, Sicherheitspartnerschaften. Die Reorganisation des Politikfeldes »Innere Sicherheit«, in TROTHA v. T., Ed., Politischer Wandel, Gesellschaft und Kriminalitätsdiskurse, Festschrift für Fritz Sack, Baden-Baden, Nomos, 1996, 299-319

LÜDERSSEN K., Abschaffen des Strafens?, Frankfurt/M., Suhrkamp,1995

LUHMANN N., Das Recht der Gesellschaft, Frankfurt/M., Suhrkamp, 1993

MITTELSTRASS J., Wohin geht die Wissenschaft? Über Disziplinarität, Transdisziplinarität und das Wissen in einer Leibniz-Welt, Konstanzer Blätter für Hochschulfragen, 1-2, 1989, 97-115

MOCCIA S., Aspects régressifs du système pénal italien, Déviance et société, 2, 1997, 137-163

MÜLLER J.C., Die Legitimation des Rechtes durch die Erfindung des symbolischen Rechtes, Kriminologisches Journal, 2, 1993, 82-97

NELKEN D., Ed., The Futures of Criminology, Oxford, Clarendon, 1994

OROZCO ABAD I., APONTE A.D., Combatientes, Rebeldes y Terroristas. Guerra y derecho en Colombia, Bogotá, Temis, 1993

PADOVANI T., La soave inquisizione. Osservazioni e rilievi a proposito delle nuove ipotesi di $»$ ravvedimento «, Rivista italiana di diritto e procedura penale, 24, 1991, 529-545

PEPINO L., SCATOLERO D., Vittime del delitto e vittimologia, Dei delitti e delle pene, 1, 1992,181-193

POULET I., BRION F., DUPONT A., Prévention en concentration au niveau local, Brugge, Vanden Broele, 1991

RESTA E., La certezza e la speranza. Saggio su diritto e violenza, Bari, Laterza, 1992

ROBERT Ph., Ed., Les politiques de prévention de la délinquance à l'aune de la recherche, Paris, L'Harmattan, 1991

ROBERT Ph., Les chercheurs face aux politiques de prévention, in, ROBERT Ph., Ed., Les politiques de prévention de la délinquance à l'aune de la recherche, Paris, L'Harmattan, 1991a, 13-27

ROBERT Ph., Évaluer la prévention, Archives de politique criminelle, 16, 1994, 53-70

ROBERT Ph., RENOUARD J.M., Bilan des connaissances en France, in ROBERT Ph., Ed., Les politiques de prévention de la délinquance à l'aune de la recherche, Paris, L'Harmattan, 1991, 191-205

ROCHÉ S., Les victimes: de la communauté à l'assurance en passant par l'État, Déviance et société, 4, 1995, 355-370

RUGGIERO V., Flexibility and Intermittent Emergency in the Italian Penal System, in RUGGIERO V., RYAN M., SIM J., Ed., Western European Penal Systems. A Critical Anatomy, London, Sage, 1995, 46-70

SCATOLERO D., Vittime, insicurezza e territorio: prospettive d'azione, Dei delitti e delle pene, 2, 1992, 179-190

SCHEERER S., Zwei Thesen zur Zukunft des Gefängnisses - und acht über die Zukunft der sozialen Kontrolle, in TROTHA v. T., Ed., Politischer Wandel, Gesellschaft und Kriminalitätsdiskurse, Festschrift für Fritz Sack, Baden-Baden, Nomos, 1996, 321-334

SCHMITT C., Der Begriff des Politischen: Text von 1933 mit einem Vorwort und drei Corollarien, Berlin, Duncker \& Humblot, 1963

SCHMITT C., Verfassungslehre (1928), Berlin, Duncker \& Humblot, 1989

SCHUMANN K.F., Der Handel mit Gerechtigkeit, Frankfurt/M., Suhrkamp, 1977 
SCHÜNEMANN B., Zur Stellung des Opfers im System der Strafrechtspflege, Neue Zeitschrift für Strafrecht, 1986, 193-200

SELMINI R., Attività di prevenzione e sicurezza: il ruolo dell'ente locale, Sicurezza e territorio, 17, 1994, 9-16

SIM J., RUGGIERO V., RYAN M., Punishment in Europe: Perceptions and Commonalities, in RUGGIERO V., RYAN M., SIM J., Ed., Western European Penal Systems. A Critical Anatomy, London, Sage, 1995, 1-23

SMAUS G., Technokratische Legitimierungen des Strafrechts, Zeitschrift für Rechtssoziologie, 1985, 90-103

SMAUS, G., Das Strafrecht und die gesellschaftliche Differenzierung, Baden-Baden, Nomos, 1998

STEFANI G., LEVASSEUR G., BOULOC B., Droit pénal général, Paris, 14e ed., Dalloz, 1992

SWAANINGEN VAN R., Criminologia critica e declino del Welfare State, Dei delitti e delle pene, 3, 1994, 31-56

TETZLAFF, R. (Hg.), Menschenrechte und Entwicklung, Bonn, 1993

TOURNIER P., La prison à la lumière des nombres, Lignes, 27, 1996, 128-141

VOURC'H C., Synthèse des débats, in ROBERT Ph., Ed., Les politiques de prévention de la délinquance à l'aune de la recherche, Paris, L'Harmattan, 1991, 265-279

ZAFFARONI E.R., Sistemas penales y derechos humanos en America Latina, Buenos Aires, Depalma 1986

ZAFFARONI E.R., En busca de las penas perdidas, Buenos Aires, Ediar, 1989

ZAUBERMAN R., Victimes en France: des positions, intérêts et stratégies diverses, Déviance et société, 1, 1991, 27-49
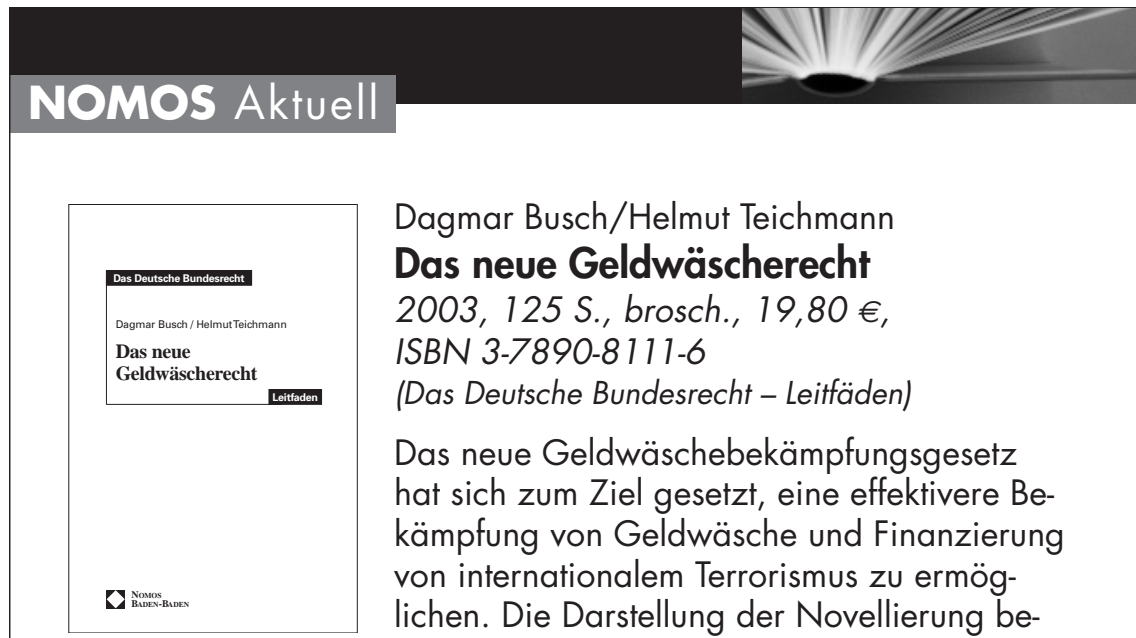

Dagmar Busch/Helmut Teichmann

Das neue Geldwäscherecht

2003, 125 S., brosch., 19,80€, ISBN 3-7890-8111-6

(Das Deutsche Bundesrecht - Leiffäden)

Das neue Geldwäschebekämpfungsgesetz hat sich zum Ziel gesetzt, eine effektivere Bekämpfung von Geldwäsche und Finanzierung von internationalem Terrorismus zu ermöglichen. Die Darstellung der Novellierung beinhaltet u.a. die Umsetzung der europäischen Geldwäsche-Richtlinie, die Ausgestaltung einer deutschen Zentralstelle für Verdachtsanzeigen, sowie die Umsetzung der bisherigen Erfahrungen mit dem alten Geldwäschegesetz.

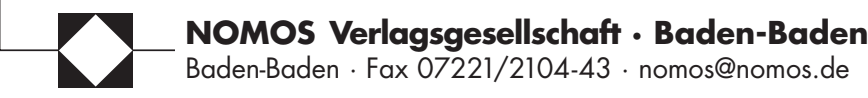

